\title{
R-Regular Integers Modulo $n^{r}$
}

\section{GaneshwarRao ${ }^{\mathrm{a}}$}

${ }^{a}$ Chaitanya Bharathi Institute of Technology, Gandipet, Hyderabad, Telangana

Article History: Received: 11 January 2021; Accepted: 27 February 2021; Published online: 5 April 2021

Abstract: Introducing the notion of ar-regular integer modulo $n^{r}$ we obtain some basic properties of such integers and arithmetic properties of certain functions related to them.

Keywords: r-regular integer modulo $n^{r}$, unitary divisor, r-free integer, r-gcd of two integers

\section{Introduction}

Let $\mathrm{r}$ be a fixed positive integer. A positive integer a is said to be r-regular modulo $n^{r}$ if there is an integer $\mathrm{x}$ such that $a^{r+1} x \equiv a^{r}\left(\bmod n^{r}\right)$. The case $r=1$ gives the notion of aregular integer moduleon, introduced by (Morgado, J, 1972; Morgado J , 1974) who made an investigation of their properties.

Clearly $a=0$ is r-regular modulo $n^{r}$ for every $n \geq 1$. Also if $a \equiv b\left(\bmod n^{r}\right)$ then a and b are r-regular modulo $n^{r}$ simultaneously. Further, if a and b are r-regular modulo $n^{r}$ then so is ab.

For positive integers $\mathrm{a}$ and $\mathrm{b}$ their greatest $\mathrm{r}^{\text {th }}$ power common divisor is denoted by $(a, b)_{r}$ and is called the $\mathrm{r}$ gcd of $a$ and $b$. Note that $(a, b)_{1}=(a, b)$, the gcd of $a$ and $b$.

We recall the notions given in (McCarthy, 1985):

A complete set of residues modulo $n^{r}$ is called a $(n, r)$-residue system. $C_{n, r}=\left\{a: 1 \leq a \leq n^{r}\right\}$ is the minimal $(n, r)$-residue system. The set of all a in an $(n, r)_{\text {-residue system such that }}\left(a, n^{r}\right)_{r}=1$ is called a reduced $(n, r)_{\text {-residue system. }} R_{n, r}=\left\{a \in C_{n, r}:\left(a, n^{r}\right)_{r}=1\right\}_{\text {is the minimal reduced }}(n, r)_{\text {-residue }}$ system.

(V.L.Klee, 1948) defined a generalization $\varphi_{r}$ of the Euler's function by $\varphi_{r}(n)=\#\left\{a: 1 \leq a \leq n\right.$ and $\left.(a, n)_{r}=1\right\}$ and proved that

$$
\varphi_{r}(n)=\sum_{d \mid n} \mu_{r}(d) \cdot \frac{n}{d}
$$

Where ${ }^{\mu}$ is the r-analogue of the Mobius function ${ }^{\mu}$ given by

$$
\mu_{r}(n)= \begin{cases}1 & \text { if } n=1 \\ (-1)^{t} & \text { if } n=\left(p_{1} p_{2} \ldots p_{t}\right)^{r} \text { where } p_{1}<p_{2}<\ldots<p_{t} \text { are primes } \\ 0 & \text { otherwise }\end{cases}
$$


Note that $\mu_{1}=\mu$ and that $\varphi_{r}\left(n^{r}\right)=\# R_{n, r}$.

Let $\operatorname{Reg}_{r}(n)=\left\{a \in C_{n, r}: a\right.$ is r-regular modulo $\left.n^{r}\right\}$ and $\rho_{r}\left(n^{r}\right)=\# \operatorname{Reg}_{r}(n)$.

Observe that any $a \in R_{n, r}$ is in $\operatorname{Reg}_{r}(n) ._{\text {In fact, if }} a \in R_{n, r}$ then $\left(a, n^{r}\right)_{r}=1$ so that $\left(a, n^{r}\right)=1$ and therefore there is an integer $x_{0}$ such that $a x_{0} \equiv 1\left(\bmod n^{r}\right)$ which gives $a^{r+1} x_{0} \equiv a^{r}\left(\bmod n^{r}\right)$ showing $a \in \operatorname{Reg}_{r}(n)$. Hence $\varphi_{r}\left(n^{r}\right)<\rho_{r}\left(n^{r}\right) \leq n^{r}$ for every $n>1$, with $\rho_{r}\left(n^{r}\right)=n^{r}$ if and only if n is squarefree.

Recently (Laszlo Toth, 2008; Yokesh, T.L., 2020) has studied several properties of the function $\rho(n):=\rho_{1}(n)$.

In this paper we prove some basic properties of the integers in the set $\operatorname{Reg}_{r}(n)$ and certain arithmetic properties of the function $\rho_{r}\left(n^{r}\right)$

\section{Integers in $\operatorname{Reg}_{\mathrm{r}}(n)$}

In all that follows $n>1$ be of the canonical form:

$$
n=p_{1}^{\alpha_{1}} p_{2}^{\alpha_{2}} \ldots p_{t}^{\alpha_{t}}
$$

where $p_{1}<p_{2}<\ldots<p_{t}$ are primes and $\alpha_{i}$ are integers $\geq 1$.

Theorem 1.For an integer $a \geq 1$ the following are equivalent:

${ }_{1.1} a \in \operatorname{Reg}_{r}(n)$

1.2 for every $i \in\{1,2, \ldots, t\}$ we have either $p_{i} \nmid a_{\text {or }} p_{i}^{\alpha_{i} r} \mid a^{r}$

1.3 $\left(a, n^{r}\right)_{r}\left\|n^{r}, d\right\| m_{\text {means that }} d \mid m$ and $\left(d, \frac{m}{d}\right)=1, \quad$ in which case $\mathrm{d}$ is called a unitary divisor of m)

$$
a^{\varphi_{r}\left(n^{r}\right)+r} \equiv a^{r}\left(\bmod n^{r}\right)
$$

1.5 There is an integer $k \geq 1$ such that $a^{k+r} \equiv a^{r}\left(\bmod n^{r}\right)$.

Proof: $\quad$ Suppose $a \in \operatorname{Reg}_{r}(n)$ so that $a^{r+1} x_{0} \equiv a^{r}\left(\bmod n^{r}\right)$ for some integer $x_{0}$. Therefore for each $i(1 \leq i \leq t), p_{i}^{\alpha_{i} r} \mid a^{r}\left(a x_{0}-1\right)$. Since $\left(a, a x_{0}-1\right)=1$ we have $\left(a^{r}, a x_{0}-1\right)=1$, we have either $p_{i} \nmid a_{\text {or }} p_{i} \mid a^{r}$ for each $i$, and in the latter case it follows $p_{i}^{\alpha_{i} r} \mid a^{r}$. Thus (i) $\Rightarrow$ (ii).

Assume (ii). That is, a is an integer $\geq 1$ such that either $p_{i} \nmid a_{\text {or }} p_{i}^{\alpha_{i} r} \mid a^{r} \cdot$ We have to show $a \in \operatorname{Reg}_{r}(n)$.

In case $p_{i} \nmid a$ then $\left(a, p_{i}^{\alpha_{i} r}\right)=1$ so that there is an integer $x_{i}$ with $a x_{i} \equiv 1\left(\bmod p_{i}^{\alpha_{i} r}\right)$ and hence $a^{r+1} x_{i} \equiv a^{r}\left(\bmod p_{i}^{\alpha_{i} r}\right)$. 
In case $p_{i}^{\alpha_{i} r} \mid a^{r}$ then for any integer $x, a^{r+1} x \equiv a^{r}\left(\bmod p_{i}^{\alpha_{i} r}\right)$ holds. Thus $a^{r+1} x \equiv a^{r}\left(\bmod p_{i}^{\alpha_{i} r}\right)$ is solvable for $1 \leq i \leq t$ and hence $a^{r+1} x \equiv a^{r}\left(\bmod p_{1}^{\alpha_{1} r} \cdot p_{2}^{\alpha_{2} r} \ldots . p_{t}^{\alpha_{t} r}\right)$ is solvable, showing $a \in \operatorname{Reg}_{r}(n)$. Thus (ii) $\Rightarrow$ (i).

Note that (ii) holds $\Leftrightarrow a^{r}=a_{0} \cdot d^{r}$, where $d^{r}=\prod_{p_{i} \mid a} p_{i}^{\alpha_{i} r}$ and $\left(a_{0}, n\right)=1$

$$
\begin{aligned}
& \Leftrightarrow\left(a^{r}, n^{r}\right)=d^{r}, \text { which is a unitary divisor of } n^{r} \\
& \Leftrightarrow\left(a, n^{r}\right)_{r}=d^{r} \| n^{r},{ }_{\text {since }}\left(a^{r}, n^{r}\right)=\left(a, n^{r}\right)_{r} \text { Thus }_{\text {Tii) }} \Leftrightarrow \text { (iii). }
\end{aligned}
$$

(ii) $\Rightarrow$ (iv). ${ }_{\text {If }} p_{i}^{\alpha_{i} r} \mid a^{r} a_{\text {then }}^{\varphi_{r}\left(n^{r}\right)+r} \equiv a^{r}\left(\bmod n^{r}\right)$ is obvious. If $p_{i} \nmid a$, then by Euler-Fermat Theorem, $a^{\varphi\left(p_{i}^{\alpha_{i} r}\right)} \equiv 1\left(\bmod p_{i}^{\alpha_{i} r}\right)$ so that

$$
a^{\varphi_{r}\left(n^{r}\right)}=\left[a^{\varphi\left(p_{i}^{\alpha_{i} r}\right)}\right]^{\varphi_{r}\left(n^{r}\right) / \varphi\left(p_{i}^{\alpha_{i} r}\right)} \equiv 1\left(\bmod p_{i}^{\alpha_{i} r}\right),
$$

since

$$
\begin{aligned}
& m:=\frac{\varphi_{r}\left(n^{r}\right)}{\varphi\left(p_{i}^{\alpha_{i} r}\right)}=\frac{\varphi_{r}\left(p_{1}^{\alpha_{1} r}\right) \varphi_{r}\left(p_{2}{ }^{\alpha_{2} r}\right) \ldots \varphi_{r}\left(p_{t}^{\alpha_{t} r}\right)}{\varphi\left(p_{i}^{\alpha_{i} r}\right)}=\left(\prod_{j \neq i} \varphi_{r}\left(p_{j}{ }^{\alpha_{j} r}\right)\right) \frac{\varphi_{r}\left(p_{i}^{\alpha_{i} r}\right)}{\varphi\left(p_{i}^{\alpha_{i} r}\right)} \\
& =\left(1+p_{i}+\ldots+p_{i}^{r-1}\right) . \prod_{\text {Mwhere }} M=\prod_{j \neq i} \varphi_{r}\left(p_{j}^{\alpha_{j} r}\right) \text { so that mis an integer. } \\
& a_{\text {Thus }}^{\varphi_{r}\left(n^{r}\right)+r} \equiv a^{r}\left(\bmod p_{i}^{\alpha_{i} r}\right) \text { for } 1 \leq i \leq t, \text { giving (iv) }
\end{aligned}
$$

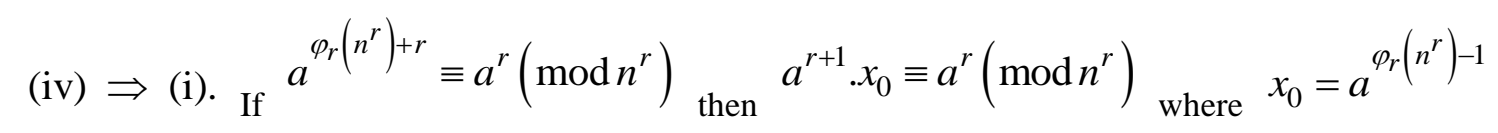
showing $a \in \operatorname{Reg}_{r}(n)$.

$$
\begin{gathered}
\text { (iv) } \Rightarrow(\mathrm{v})_{\text {is immediate with }} k=\varphi_{r}\left(n^{r}\right) . \quad \text { Also if } a^{k+r} \equiv a^{r}\left(\bmod n^{r}\right) \text { for some } k \geq 1 \text { implies } \\
a^{r+1} \cdot x_{0} \equiv a^{r}\left(\bmod n^{r}\right),{ }_{\text {where }} x_{0}=a^{k-1}, \text { showing } \\
a \in \operatorname{Reg}_{r}(n) .
\end{gathered}
$$

3. The Function $\rho_{r}\left(n^{r}\right)$.

In this section we study the function $\rho_{r}\left(n^{r}\right)$ and its relation with $\varphi_{r}\left(n^{r}\right)$. Also we express the sum $S_{r}(n)$ of the r-regular integers modulo $n^{r}$ in terms of $\rho_{r}\left(n^{r}\right)$ 
Theorem 2: For every $n \geq 1$,

$$
\rho_{r}\left(n^{r}\right)=\sum_{d^{r} \| n^{r}} \varphi_{r}\left(d^{r}\right)
$$

The function $\rho_{r}\left(n^{r}\right)$ is multiplicative and $\rho_{r}\left(p^{\alpha r}\right)=p^{\alpha r}-p^{(\alpha-1) r}+1$, for any prime pand integer $\alpha \geq 1$.

Proof: We give two proofs for the first part.

First Proof: Let $a \in \operatorname{Reg}_{r}(n)$.

If $p_{i} \nmid a$ for $1 \leq i \leq t$ then $(a, n)=1$ so that $\left(a, n^{r}\right)_{r}=\left(a^{r}, n^{r}\right)=1$ and the number of such as $\varphi_{r}\left(n^{r}\right)$.

Suppose $^{p_{i}^{\alpha_{i} r} \mid a^{r}}$ for exactly one i so that $\left(a, p_{j}\right)=1$ for $j \neq i$ and $a=b \cdot p_{i}^{\alpha_{i} r}$ where $1 \leq b \leq \frac{n^{r}}{p_{i}^{\alpha_{i} r}}$ and $\left(b, \frac{n^{r}}{p_{i}^{\alpha_{i} r}}\right)=1$; the number of such a's is $\varphi_{r}\left(\frac{n^{r}}{p_{i}^{\alpha_{i} r}}\right)$.

Suppose $p_{i}^{\alpha_{i} r} \mid a^{r}$ and $p_{j}^{\alpha_{j} r} \mid a^{r} \quad$ for $\quad 1 \leq i<j \leq t ;$ and for $k \notin\{i, j\} \quad\left(p_{k}, a\right)=1$.

Then $a=C \cdot p_{i}^{\alpha_{i} r} \cdot p_{j}^{\alpha_{j} r}, \quad 1 \leq C \leq \frac{n^{r}}{p_{i}^{\alpha_{i} r} p_{j}^{\alpha_{j} r}}$ and $\left(C, \frac{n^{r}}{p_{i}^{\alpha_{i} r} p_{j}{ }^{\alpha_{j} r}}\right)=1$; $\varphi_{r}\left(\frac{n^{r}}{p_{i}^{\alpha_{i}{ }^{r}} \cdot p_{j}^{\alpha^{r}}}\right)$;

$$
\begin{aligned}
& \qquad \rho_{r}\left(n^{r}\right)=\varphi_{r}\left(n^{r}\right)+\sum_{1 \leq i \leq t} \varphi_{r}\left(\frac{n^{r}}{p_{i}^{\alpha_{i} r}}\right)+\sum_{1 \leq i<j \leq t} \varphi_{r}\left(\frac{n^{r}}{p_{i}^{\alpha_{i} r} p_{j}^{\alpha_{j} r}}\right)+\ldots+\varphi_{r}\left(\frac{n^{r}}{p_{i}^{\alpha_{i} r} p_{2}^{\alpha_{2} r} \ldots p_{t}^{\alpha_{t} r}}\right) \\
& =y+\sum_{1 \leq i \leq t} \frac{y}{y_{i}}+\sum_{1 \leq i<j \leq t} \frac{y}{y_{i} y_{j}}+\ldots+\frac{y}{y_{1} y_{2} \ldots y_{t}} \\
& y_{i}=\varphi_{r}\left(p_{i}^{\alpha_{i} r}\right) \text { and } y=y_{1} y_{2} \ldots y_{t} .
\end{aligned}
$$

Therefore

$$
\begin{aligned}
& \rho_{r}\left(n^{r}\right)=\left(y_{1}+1\right)\left(y_{2}+1\right) \ldots\left(y_{t}+1\right) \\
& =\left(\varphi_{r}\left(p_{1}^{\alpha_{1} r}\right)+1\right)\left(\varphi_{r}\left(p_{2}^{\alpha_{2} r}\right)+1\right) \ldots\left(\varphi_{r}\left(p_{t}^{\alpha_{t} r}\right)+1\right) \\
& =\sum_{d^{r} \| n^{r}} \varphi_{r}\left(\frac{n^{r}}{d^{r}}\right)=\sum_{d^{r} \| n^{r}} \varphi_{r}\left(d^{r}\right) .
\end{aligned}
$$


Second Proof: Groupthe integers $a \in C_{n, r}$ according to the value $\left(a, n^{r}\right)_{r}=d^{r}$.

Note that $\left(a, n^{r}\right)_{r}=d^{r} \Leftrightarrow a=j . d^{r} \quad 1 \leq j \leq \frac{n^{r}}{d^{r}}$ and $\left(j, \frac{n^{r}}{d^{r}}\right)_{r}=1$.

${ }_{\text {in }} C_{n, r \text { with }}\left(a, n^{r}\right)_{r}=d^{r} \varphi_{r}\left(\frac{n^{r}}{d^{r}}\right)$. Thus $\rho_{r}\left(n^{r}\right)=\sum_{d^{r} \| n^{r}} \varphi_{r}\left(\frac{n^{r}}{d^{r}}\right)=\sum_{d^{r} \| n^{r}} \varphi_{r}\left(d^{r}\right)$.

Now

$$
\rho_{r}\left(n^{r}\right)=\sum_{D \| n^{r}} \varphi_{r}(D) \cdot \chi_{r}(D)
$$

where $\chi_{r}(m)=1$ or 0 according as $m$ is therth power of an integer or not.

Therefore $\rho_{r}\left(n^{r}\right)=\left(\varphi_{r} \chi_{r} \circ I\right)\left(n^{r}\right)$, where $I(n) \equiv 1$ for all $\mathrm{n}$ and $\circ$ is the unitary convolution of arithmetic functions discussed by (Eckford Cohen, 1960). Since unitary convolution preserves multiplicativity, we get

$\rho_{r}\left(n^{r}\right)$ is multiplicative, because $\varphi_{r}, \chi_{r}$ and I are all multiplicative.

Also $\rho_{r}\left(p^{\alpha r}\right)=\varphi_{r}\left(p^{\alpha r}\right)+1=p^{\alpha r}-p^{(\alpha-1) r}+1$, completing the proof of Theorem B.

Theorem 3. $\left(a, n^{r}\right)_{r}=1$

$$
\sum_{a \in C_{n, r}} a=\frac{1}{2} n^{r} \cdot \varphi_{r}\left(n^{r}\right)
$$

Proof: First observe that for positive integers a and b, $(a, b)_{r}=1$ if and only if $(a, b)$ is r-free (Recall that an integer not divisible by the rth power of any prime is said to be r-free). Let $q_{r}(m)=1$ or 0 according as $\mathrm{m}$ is $\mathrm{r}$-free or not. Then it is well-known (Apostol, 1998, problem 6, p.47; Ranjeeth 2020) that

$$
q_{r}(m)=\sum_{t^{r} \mid m} \mu(t)
$$

Where ${ }^{\mu}$ is the Mobius function

Now, by (4) and (1), we get

$$
\sum_{\substack{a \in C_{n, r} \\\left(a, n^{r}\right)_{r}=1}} a=\sum_{1 \leq a \leq n^{r}} a \cdot q_{r}\left(\left(a, n^{r}\right)\right)
$$




$$
\begin{aligned}
& =\sum_{1 \leq a \leq n^{r}} a\left\{\sum_{\substack{t^{r} s=a \\
t^{r} \mid n^{r}}} \mu(t)\right\} \\
& =\sum_{\substack{t^{r} s \leq n^{r} \\
t^{r} \mid n^{r}}} t^{r} s \mu(t) \\
& =\sum_{t^{r} \mid n^{r}} \mu(t) t^{r}\left\{\sum_{\substack{s \leq \frac{n^{r}}{t^{r}} \\
r}} s\right\} \\
& =\sum_{t^{r} \mid n^{r}} \mu(t) t^{r} \cdot \frac{1}{2} \cdot \frac{n^{r}}{t^{r}}\left(\frac{n^{r}}{t^{r}}+1\right) \\
& =\frac{n^{r}}{2} \sum_{t^{r} \mid n^{r}} \mu(t) \frac{n^{r}}{t^{r}}+\frac{n^{r}}{2} \sum_{t^{r} \mid n^{r}} \mu(t) \\
& =\frac{n^{r}}{2} \cdot \sum_{t_{\circ} \mid n^{r}} \mu\left(t_{\circ}\right) \frac{n^{r}}{t_{\circ}}+\frac{n^{r}}{2} \sum_{t^{r} \mid n^{r}} \mu(t) \\
& =\frac{n^{r}}{2} \cdot \varphi_{r}\left(n^{r}\right), \\
& \sum_{\text {since }} \sum_{\left.t^{r}\right|_{n}{ }^{r}} \mu(t)=0
\end{aligned}
$$

Remark 1.The case $r=1$ of Theorem $\mathrm{C}$ is the well-known formula:

$$
\sum_{\substack{1 \leq a \leq n \\(a, n)=1}} a=\frac{n \varphi(n)}{2} \text { for } n>1 \text {. (For example see (Apostol, 1998, Problem 16, p.48) }
$$

Theorem 4. If $S_{r}(n):=\sum_{a \in \operatorname{Reg}_{r(n)}} a$ then $S_{r}(n)=\frac{1}{2}\left[\rho_{r}\left(n^{r}\right)+1\right]$ for $n \geq 1$.

Proof: We have, by Theorem A, that $a \in \operatorname{Reg}_{r}(n) \Leftrightarrow\left(a, n^{r}\right)_{r}=d^{r} \| n^{r}$.

\section{Therefore}




$$
\begin{gathered}
S_{r}(n)=\sum_{\substack{a \in C_{r}, n \\
\left(a, n^{r}\right)_{r} \|^{r}}} a=\sum_{d^{r} \| n^{r}} \sum_{\substack{\left(a, n^{r}\right)_{r}=d^{r} \\
a \in C_{r, n}}} a \\
=\sum_{d^{r} \| n^{r}} d^{r} \sum_{\substack{j \in C \\
\frac{n^{r}}{d^{r}, r} \\
\left(j, \frac{n^{r}}{d^{r}}\right)_{r}=1}} j, \text { Since } \\
\text { where }
\end{gathered}
$$

Now, in view of Theorem $\mathrm{C}$ and Theorem $\mathrm{B}$, for $n \geq 1$ we have

$$
\begin{aligned}
& S_{r}(n)=n^{r}+\sum_{\substack{d^{r} \| n^{r} \\
d^{r}<n^{r}}} d^{r} \cdot \frac{1}{2} \cdot \frac{n^{r}}{d^{r}} \cdot \varphi^{r}\left(\frac{n^{r}}{d^{r}}\right) \\
& =n^{r}+\frac{n^{r}}{2} \sum_{d^{r} \| n^{r}} \varphi^{r}\left(\frac{n^{r}}{d^{r}}\right) \\
& =n^{r}+\frac{n^{r}}{2} \cdot\left[\rho_{r}\left(n^{r}\right)-1\right] \\
& =\frac{n^{r}}{2}\left[\rho_{r}\left(n^{r}\right)+1\right],
\end{aligned}
$$

proving the theorem.

\section{References}

1. Apostol, Tom M., (1998) Introduction to Analytic Number Theory, Springer International Student Edition, Naroso Publishing House, New Delhi, 1998.

2. Eckford Cohen, (1960)Arithmetical Functions Associated with the Unitary Divisors of an Integer, Math. Zeitschr. 74, 66-80.

3. Klee, V. L, (1948)Generalization of Euler's Function, Amer. Math., Monthly, 55, 358-359.

4. Laszlo Toth, Regular Integers Modulo n, (2008) AnnalsUniv. Sci. Budapest., Sect. Comp. 29, 263-275.

5. McCarthy, Paul J., (1985). Introduction to Arithmetical Functions, Springer-Verlag, New York.

6. Morgado, J, (1972). InteiorsRegulares Modulo n, Gazeta de Mathematical (Lisboa), 33,.125-125, 1-5.

7. Morgado, J. (1974)A Property of the Euler $\varphi$-Function Concerning the Integers which are Regular Modulo n, Portugal. Math., 33, 185-191.

8. Ranjeeth, S., Latchoumi, T. P., \& Paul, P. V. (2020). Role of gender on academic performance based on different parameters: Data from secondary school education. Data in brief, 29, 105257.

9. Yookesh, T. L., Boobalan, E. D., \& Latchoumi, T. P. (2020, March). Variational Iteration Method to Deal with Time Delay Differential Equations under Uncertainty Conditions. In 2020 International Conference on Emerging Smart Computing and Informatics (ESCI) (pp. 252-256). IEEE. 